\title{
LETTER
}

\section{SARS-CoV-2 viral loads and serum lgA/lgG immune responses in critically ill COVID-19 patients}

\author{
Slim Fourati 1,2,3, Sophie Hue 2,4,5,6, Jean-Michel Pawlotsky ${ }^{1,2,3}$, Armand Mekontso-Dessap 2,7,8 \\ and Nicolas de Prost ${ }^{2,7,8,9^{*}}$ (B)
}

(c) 2020 Springer-Verlag GmbH Germany, part of Springer Nature

\section{Dear Editor}

The pandemic of coronavirus disease 2019 (COVID19) caused by the novel severe acute respiratory syndrome coronavirus 2 (SARS-CoV-2) represents the greatest global public health crisis that occurred during the last decades. Among hospitalized patients, up to $25 \%$ will develop acute respiratory failure and the acute respiratory distress syndrome (ARDS) and require intensive care unit (ICU) admission. The median duration between onset of symptoms and ICU admission ranges from 7 to 12 days [1], suggesting a gradual deterioration in the majority of cases. Although the clinical characteristics of patients requiring ICU admission have now been well described [2-4], their viro-immunological features are still unknown. Whether a higher titer of SARS-CoV2 -specific antibodies may reduce viral RNA load in upper respiratory samples and eventually mitigate the course of infection in patients admitted in the ICU has not been established. We examined the relationship between SARS-CoV-2 viral loads collected from nasopharyngeal swabs on ICU admission, concomitant SARS-CoV-2-specific IgA and IgG antibody titers, and day- 28 mortality.

This is a prospective monocenter study, which included all patients diagnosed with RT-PCR-confirmed SARS$\mathrm{CoV}-2$ infection consecutively admitted in the medical ICU at Henri Mondor Hospital, Créteil, France, between March 8, 2020, and March 26, 2020. The study has received the approbation of an institutional review

\footnotetext{
*Correspondence: nicolas.de-prost@aphp.fr

${ }^{9}$ Service de Médecine Intensive Réanimation, Hôpital Henri Mondor Créteil, France

Full author information is available at the end of the article

Slim Fourati and Sophie Hue have contributed equally to this work.
}

board (Comité de Protection des Personnes Ile de France II; reference number: 3675-NI). Informed consent was obtained from all patients or their relatives. Nasopharyngeal swabs and sera were collected from patients within $48 \mathrm{~h}$ of ICU admission. The cycle threshold values of RTPCR were used as indicators of the viral load of SARSCoV-2 RNA in nasopharyngeal specimens, with lower cycle threshold values corresponding to higher viral load. IgA and IgG antibodies against SARS-CoV-2 spike protein subunit 1 (S1) were quantified in patients' serum using ELISAs (Euroimmun Medizinische Labordiagnostika, Lübeck, Germany) and expressed in arbitrary units (AU). The primary clinical outcome was day-28 mortality.

Twenty-five patients [mean age $60 \pm 14$ years; males $80 \%(n=20 / 25)]$ were admitted in the ICU for severe SARS-CoV-2 infection during the study period. The median time elapsed between the first symptoms and ICU admission was 9 days [6-12]. Invasive mechanical ventilation was required in $96 \%(n=24 / 25)$ of patients during ICU stay, and the mortality at day- 28 of ICU admission was $32 \%(n=8 / 25) \quad$ (Supplemental Table 1). There was a significant correlation between the time elapsed between the first symptoms and ICU admission and the titer of both anti-S1 IgA (Spearman's $r=0.70$; $p<0.001)$ and anti-S1 IgG $(r=0.68 ; p<0.001)$ measured in sera obtained upon ICU admission (Fig. 1a, b). No correlation was found between the absolute counts of peripheral $\mathrm{B}$ or $\mathrm{T}$ lymphocytes and the titers of anti-S1 IgA $(r=0.002, p=0.99$ and $r=0.10, p=0.624$, respectively) or IgG $(r=0.12, p=0.56$, and $r=0.13, p=0.53$, respectively). There was also no correlation between peripheral $\mathrm{B}$ or $\mathrm{T}$ lymphocyte counts and viral loads ( $r=0.34, p=0.093$, and $r=0.24, p=0.247$, respectively).

\section{实 Springer}



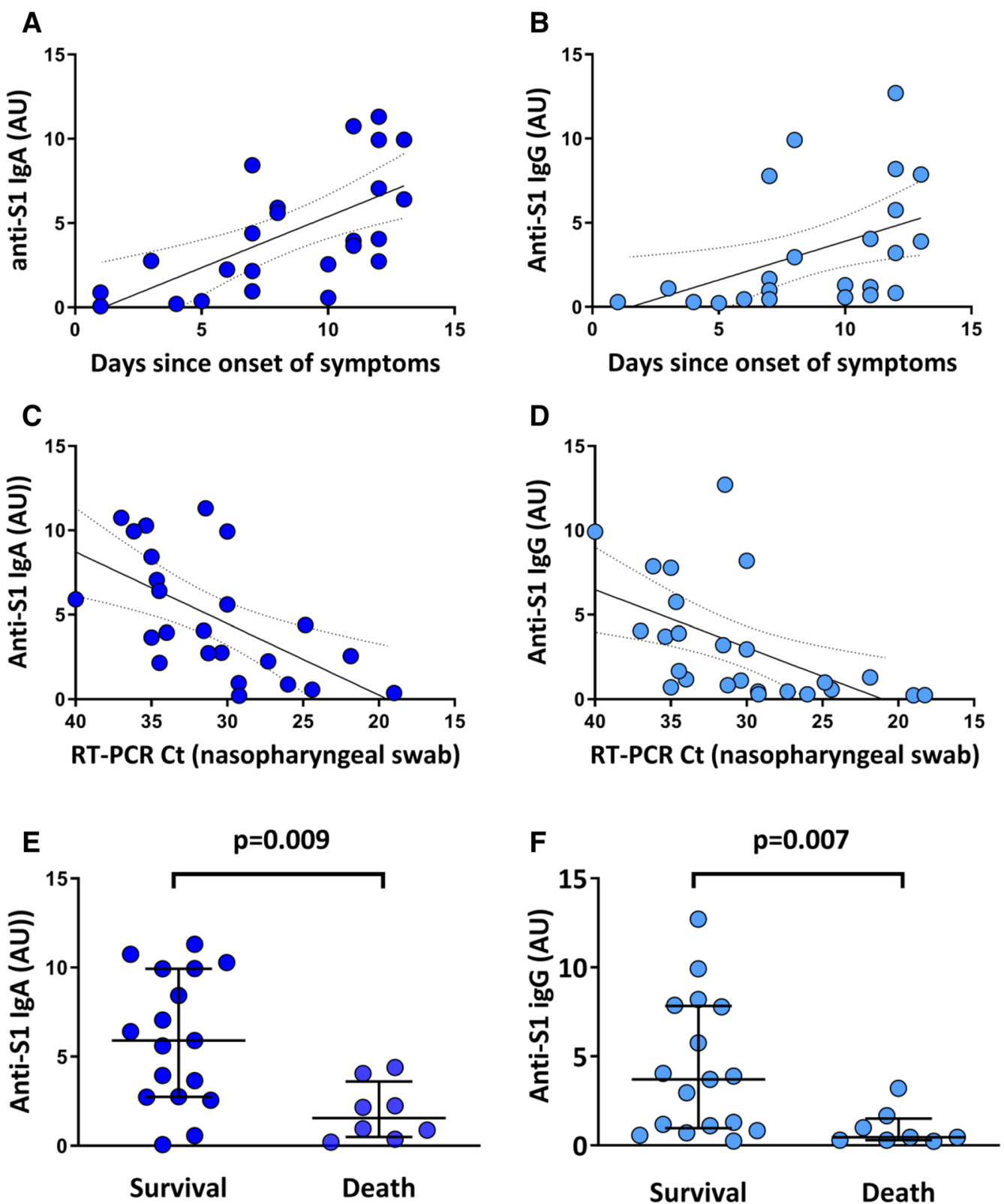

Fig. 1 a Correlation between the number of days since onset of symptoms and intensive care unit (ICU) admission and the serum titer of anti-S1 $\lg \mathrm{A}$, expressed in arbitrary units (AU) (Spearman's $r=0.70 ; p<0.001 ; r^{2}=0.40$ ); $\mathbf{b}$ correlation between the number of days since onset of symptoms of SARS-CoV-2 infection and ICU admission and the serum titer of anti-S1 $\mathrm{lgG}(\mathrm{AU})$ (Spearman's $r=0.68 ; p<0.001, r^{2}=0.22$ ); c correlation between the serum titer of anti-S1 lgA (AU) and the SARS-CoV-2 viral load measured in nasopharyngeal swabs (expressed in cycle threshold value, Ct) (Spearman's $\left.r=0.69 ; p<0.001 ; r^{2}=0.35\right)$; $\mathbf{d}$ correlation between the serum titer of anti-S1 lgG (AU) and the SARS-CoV-2 viral load measured in nasopharyngeal swabs (expressed in Ct) (Spearman's $r=0.72 ; p<0.0001 ; r^{2}=0.29$ ); note that the $x$-axis of $\mathbf{c}$, $\mathbf{d}$ is inverted so as to reflect that the RT-PCR Ct is inversely correlated with RNA viral load; e comparisons of serum anti-S1 IgA titers obtained upon ICU admission between patients who were alive at day-28 of ICU admission and patients who died; $\mathbf{f}$ comparisons of serum anti-S1 IgG titers obtained upon ICU admission between patients who were alive at day 28 of ICU admission and patients who died; continuous lines represent the lines of best fit of the linear regressions and the dotted lines show their $95 \%$ confidence intervals; horizontal lines represent the median value; $p$ values displayed in $\mathbf{e}$, $\mathbf{f}$ come from the Mann-Whitney test

We observed an inverse correlation between the viral load obtained from nasopharyngeal swabs and the serum level of anti-S1 IgA $(r=0.69 ; p<0.001)$ or $\operatorname{IgG}(r=0.72$; $p<0.0001$ ) (Fig. 1c, d). Finally, we explored the relationship between IgA/G titers measured upon ICU admission and day-28 mortality. As shown in Fig. 1e, f, patients 
who were still alive at day-28 displayed significantly higher titers of anti-S1 IgA or IgG upon admission than those who had died at day-28. Additionally, the serum titer of anti-S1 IgA was a protective factor of day-28 mortality, even after adjusting for SOFA (adjusted odds ratio $(\mathrm{aOR})=0.45[0.21-0.98] ; p=0.045)$ or age $(\mathrm{aOR}=0.56$ [0.33-0.95]; $p=0.032$ ) (Supplemental Table 2), two important determinants of outcome during severe SARS$\mathrm{CoV}-2$ infections.

Our study certainly has some limitations, related to its monocenter design as well as to the small number of patients included, limiting our ability to adjust the observed relationship between anti-S1 IgA/G titers and day-28 mortality for potential confounders. Yet, our results suggest that the absence of early humoral response in a subset of severe patients is associated with a higher viral load in the upper respiratory tract upon admission and might have a deleterious impact on survival.

\section{Electronic supplementary material}

The online version of this article (https://doi.org/10.1007/s00134-020-06157-5) contains supplementary material, which is available to authorized users.

\begin{abstract}
Author details
${ }^{1}$ Virology Unit, Département de prévention, diagnostic et traitement des infections, Hôpitaux Universitaires Henri Mondor, Assistance Publique - Hôpitaux de Paris (AP-HP), Créteil, France. ${ }^{2}$ INSERM U955, Université Paris-Est Créteil Val de Marne (UPEC), Créteil, France. ${ }^{3}$ INSERM U955 Team «Virus Hepatology Cancer », Créteil, France. ${ }^{4}$ Département Immunologie-Hématologie, Hôpitaux Universitaires Henri Mondor, Assistance Publique - Hôpitaux de Paris (AP-HP), Créteil, France. ${ }^{5}$ INSERM U955 Team 16, Créteil, France. ${ }^{6}$ Vaccine Research Institute (VRI), Faculté de Médecine, Université Paris Est Créteil, 94010 Créteil, France. ${ }^{7}$ Médecine Intensive Réanimation, Hôpitaux Universitaires Henri Mondor, Assistance Publique - Hôpitaux de Paris (AP-HP), Créteil, France. ${ }^{8}$ Groupe de Recherche Clinique CARMAS, Université Paris Est-Créteil, Créteil, France. ${ }^{9}$ Service de Médecine Intensive Réanimation, Hôpital Henri Mondor, Créteil, France.
\end{abstract}

\section{Acknowledgements}

The authors would like to thank Dr Inès Bendib, Thiziri Sadaoui, Thomas Frapart, and Simon Rivoal for helping collect clinical data, Audrey Riou, Alexandre
Soulier, and Asma Beldi-Ferchiou for performing laboratory analyses, and Prof. Marie-Hélène Delfau-Larue for critically reviewing the manuscript.

Funding

None

Compliance with ethical standards

\section{Conflicts of interest}

SF, SH, AMD, and NDP have no conflict of interest to disclose. JMP has received research grants from Abbott, Abbvie, and Gilead; he has served as an advisor for Abbott, Abbvie, Gilead, Merck, and Siemens Healthcare.

\section{Publisher's Note}

Springer Nature remains neutral with regard to jurisdictional claims in published maps and institutional affiliations.

Accepted: 9 June 2020

Published online: 22 June 2020

\section{References}

1. Phua J, Weng L, Ling L, Egi M, Lim CM, Divatia JV, Shrestha BR, Arabi YM, Ng J, Gomersall CD, Nishimura M, Koh Y, Du B (2020) Intensive care management of coronavirus disease 2020 (COVID-19): challenges and recommendations. Lancet Respir Med 8(5):506-517

2. Wu C, Chen X, Cai Y, Xia J, Zhou X, Xu S, Huang H, Zhang L, Du C, Zhang Y, Song J, Wang S, Chao Y, Yang Z, Xu J, Chen D, Xiong W, Xu L, Zhou F, Jiang J, Bai C, Zheng J, Song Y (2020) Risk factors associated with acute respiratory distress syndrome and death in patients with coronavirus disease 2019 pneumonia in Wuhan, China. JAMA Intern Med. https://doi. org/10.1001/jamainternmed.2020.0994

3. Grasselli G, Zangrillo A, Zanella A, Antonelli M, Cabrini L, Castelli A, Cereda D, Coluccello A, Foti G, Fumagalli R, lotti G, Latronico N, Lorini L, Merler S, Natalini G, Piatti A, Ranieri MV, Scandroglio AM, Storti E, Cecconi M, Pesenti A (2020) Baseline characteristics and outcomes of 1591 patients infected with SARS-CoV-2 admitted to ICUs of the Lombardy Region, Italy. JAMA 323(16):1574-1581

4. Yang X, Yu Y, Xu J, Shu H, Xia J, Liu H, Wu Y, Zhang L, Yu Z, Fang M, Yu T, Wang Y, Pan S, Zou X, Yuan S, Shang Y (2020) Clinical course and outcomes of critically ill patients with SARS-CoV-2 pneumonia in Wuhan, China: a single-centered, retrospective, observational study. Lancet Respir Med 8(5):475-481 